\title{
CXCR4-mediated osteosarcoma growth and pulmonary metastasis is promoted by mesenchymal stem cells through VEGF
}

\author{
PENG ZHANG ${ }^{1,2 *}$, LING DONG ${ }^{3 *}$, KANG YAN $^{1}$, HUA LONG $^{1}$, TONG-TAO YANG ${ }^{1}$, \\ MING-QING DONG ${ }^{4}$, YONG ZHOU ${ }^{1}$, QING-YU FAN ${ }^{1}$ and BAO-AN MA ${ }^{1}$ \\ ${ }^{1}$ Department of Orthopedic Surgery, Tangdu Hospital, Fourth Military Medical University, Xi'an 710038; \\ ${ }^{2}$ Department of Orthopedic Surgery, Urumqi General Hospital, Urumqi 830000; Departments of ${ }^{3}$ Physiology, \\ ${ }^{4}$ Pathology and Pathophysiology, Fourth Military Medical University, Xi'an 710032, P.R. China
}

Received February 14, 2013; Accepted April 19, 2013

DOI: $10.3892 /$ or.2013.2619

\begin{abstract}
Chemokines and chemokine receptor 4 (CXCR4) play an important role in metastasis. CXCR4 is also expressed in the human osteosarcoma cell line 9607-F5M2 (F5M2), which has a high tumorigenic ability and potential for spontaneous pulmonary metastasis. Mesenchymal stem cells (MSCs) contribute to the formation of the tumor stroma and promote metastasis. However, mechanisms underlying the promotion of osteosarcoma growth and pulmonary metastasis by MSCs are still elusive. Our study co-injected the human MSCs and F5M2 cells into the caudal vein of nude mice. The total number of tumor nodules per lung was significantly increased in the F5M2+MSC group compared to the other groups (control, F5M2 cells alone and MSCs alone) at week six. Moreover, a high number of Dil-labeled MSCs was present also at the osteosarcoma metastasis sites in the lung. Using Transwell assays, we found that F5M2 cells migrate towards MSCs, while the CXCR4 inhibitor AMD3100 decreased the migration potential of F5M2 cells towards MSCs. Furthermore, upon treatment with F5M2-conditioned medium, MSCs expressed and secreted higher levels of VEGF as determined by immunohistochemistry, western blotting
\end{abstract}

Correspondence to: Professor Bao-An Ma or Professor Qing-Yu Fan, Department of Orthopedic Surgery, Tangdu Hospital, Fourth Military Medical University, Xi'an, Shaanxi 710038, P.R. China

E-mail: gukemba@fmmu.edu.cn

E-mail: bonetm@fmmu.edu.cn

*Contributed equally

Abbreviations: CM, conditioned medium; CM-Dil, Dil chloromethyl-dialkylcarbocyanine; CXCR, chemokines and chemokine receptors; MSCs, mesenchymal stem cells; VEGF, vascular endothelial growth factor; VEGF Ab, anti-VEGF antibody; SDF-1, stromal cell-derived factor 1; SDF-1 Ab, human SDF-1 antibody

Key words: osteosarcoma, mesenchymal stem cells, VEGF, CXCR4, metastasis and ELISA, respectively. Importantly, co-cultured with F5M2 cells, MSCs expressed and secreted higher VEGF levels, while AMD3100 dramatically decreased the VEGF secretion by MSCs. However, CXCR4 expression on F5M2 cells was not significantly increased in the co-culture system. Additionally, VEGF increased the proliferation of both MSCs and F5M2 cells. These findings suggest that CXCR4-mediated osteosarcoma growth and pulmonary metastasis are promoted by MSCs through VEGF.

\section{Introduction}

Osteosarcoma accounts for $\sim 15 \%$ of all primary bone tumor cases (1). Approximately $95 \%$ of the patients who died of metastatic disease exhibited metastases in the lungs, as indicated in autopsy (2). The mechanisms underlying the development, progression and metastasis of osteosarcoma remain elusive. We previously established the osteosarcoma cell line, 9607-F5M2 (F5M2), which has a high tumorigenic ability and potential for spontaneous pulmonary metastasis (3).

Non-hematopoietic mesenchymal stem cells (MSCs) in the bone marrow are characterized by clonal, plastic adherent cells capable of differentiating into osteoblasts, adipocytes and chondrocytes (4). MSCs are also stromal cells, structural components of the bone marrow that support in vitro hematopoiesis by providing extracellular matrix components, cytokines and growth factors (5-7). MSCs have been shown to target microscopic tumors and subsequently proliferate and differentiate, contributing to the formation of a significant portion of the tumor stroma and promote breast cancer metastasis (8). These observations indicate the complexity of the relationship between MSCs and tumors. There is, however, little data concerning the relationship between MSCs and osteosarcoma. Chemokines are small soluble molecules that are best known for their potent ability to induce cellular migration. Many types of cancer cells express chemokines and chemokine receptors (CXCR), such as CXCR4 (9). CXCR4 expression on tumor cells is upregulated by hypoxia and angiogenic factors, such as vascular endothelial growth factor (VEGF). Patients with CXCR4/VEGF positive tumors 
have a significantly shorter survival, suggesting that the CXCR4/VEGF could be a predictor of potential metastatic development (10).

In order to study the interaction and the underlying mechanism between MSCs and osteosarcoma, we co-injected MSCs and osteosarcoma cells into nude mice and monitored tumor development and progression, namely growth and pulmonary metastasis in response to MSCs. Finally, we performed in vitro experiments including Transwell assays, MTT, ELISA and protein expression analysis to study the possible mechanisms, with particular focus on the CXCR4/VEGF signaling pathway and found that CXCR4-mediated osteosarcoma growth and pulmonary metastasis were promoted by MSCs through VEGF.

\section{Materials and methods}

Isolation and culture of human MSCs and F5M2. MSCs were isolated and expanded according to a modification of a previously described protocol $(11,12)$. We identified MSCs by CD90 and CD45, in which $>95 \%$ of the population was positive for CD90 expression and $>98 \%$ negative for CD45 surface molecules. The donor of the cells was a healthy individual without metabolic or inherited disorders or other diseases that might affect the current study. A signed consent was obtained and the study was approved by the Institutional Review Board of the Fourth Military Medical University. In brief, human MSCs were isolated by density-gradient centrifugation and cultured in low-glucose Dulbecco's modified Eagle's medium (DMEM, Gibco Laboratories, USA) supplemented with $10 \%$ fetal bovine serum (FBS) (Hyclone), $100 \mathrm{U} / \mathrm{ml}$ penicillin and $100 \mu \mathrm{g} / \mathrm{ml}$ streptomycin at $37^{\circ} \mathrm{C}$, in a humidified atmosphere containing $5 \%$ carbon dioxide $\left(\mathrm{CO}_{2}\right)$. Culture medium was replaced by fresh medium twice a week. Confluent cells were harvested for passage 3-6 with $0.25 \%$ trypsin. The human osteosarcoma cell line 9607-F5M2 (F5M2) was grown in DMEM supplemented with 10\% FBS (Hyclone) (3). Conditioned medium (CM) was derived by adding $1 \%$ FBS medium to cells at $80 \%$ confluence for $24 \mathrm{~h}$.

Tumor xenografts in nude mice. Study protocols involving mice were approved by the Animal Ethics Committee of the Fourth Military Medical University. BALB/c nu/nu (nude mice) from the animal center of the Fourth Military Medical University, Xi'an, Shaanxi, China (approval ID: 2009043) (four weeks of age, male) were divided into four groups $(n=6$ animals per group): control group, MSCs group, F5M2 group and MSCs+F5M2 group. MSCs and F5M2 cells were resuspended in PBS at a final concentration of $1 \times 10^{7}$ cells $/ \mathrm{ml}$. MSCs were labeled with $4 \mu \mathrm{g} / \mathrm{ml}$ chloromethyl-dialkylcarbocyanine (CM-Dil, Dil) (Invitrogen) in pre-warmed PBS for $15 \mathrm{~min}$ at $37^{\circ} \mathrm{C}$ followed by an incubation for $15 \mathrm{~min}$ at $4^{\circ} \mathrm{C}$. Cells were washed with PBS and resuspended in PBS before they were used. MSCs and/or F5M2 cells were injected into the caudal vein of nude mice at a dose of $2 \times 10^{6}$ cells per mouse. After 6 weeks, mice were sacrificed with excess pentobarbital. The number of pulmonary matastatic tumor nodules was counted under a low-powered dissecting stereomicroscope. The lung, liver, spleen, kidney and serum were harvested for further analysis (11).
Co-culture and Transwell assays. In direct co-culture studies, MSCs were labeled with Dil $(4 \mu \mathrm{g} / \mathrm{ml})$. Then F5M2 cells and

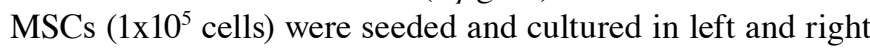
area separately and the cells had adhered after $12 \mathrm{~h}$. Cells were grown in DMEM supplemented with $1 \%$ FBS (Hyclone), $100 \mathrm{U} / \mathrm{ml}$ penicillin and $100 \mu \mathrm{g} / \mathrm{ml}$ streptomycin at $37^{\circ} \mathrm{C}$, in a humidified atmosphere with $5 \% \mathrm{CO}_{2}$ for 5 days. Then the F5M2 cells and MSCs labeled with $5 \mu \mathrm{g} / \mathrm{ml}$ Hoechst (Sigma) in pre-warmed PBS for $10 \mathrm{~min}$ at $37^{\circ} \mathrm{C}$. The cells were washed with PBS three times, then were fixed in buffered isotonic formaldehyde (100 $\mathrm{ml}$ of $37 \%$ formaldehyde solution, $900 \mathrm{ml}$ distilled water, $4 \mathrm{~g}$ monobasic sodium phosphate and $6.5 \mathrm{~g}$ dibasic sodium phosphate).

The invasive potential of the cells was measured in $6.5-\mathrm{mm}$ transwells with an $8-\mu \mathrm{m}$ pore polycarbonate membrane insert (Corning, NY), according to the manufacturer's instructions. The top chamber filter was coated with $50 \mu \mathrm{l}$ of diluted Matrigel following the standard procedure and incubated at $37^{\circ} \mathrm{C}$ for $2 \mathrm{~h}$. The lower chamber was filled with $600 \mu \mathrm{l}$ of DMEM containing $1 \%$ FBS as a chemoattractant. Cells were serumfree-starved overnight and then harvested and resuspended in migration medium (DMEM with $0.5 \% \mathrm{BSA}$ ). A suspension of 5,000 cells in $100 \mu \mathrm{l}$ migration medium was then added into each top chamber. After incubation for $16 \mathrm{~h}$, the non-invading cells that remained on the upper surface were removed with a cotton swab. The invasive cells on the lower surface of the membrane insert were fixed with $4 \%$ paraformaldehyde for $30 \mathrm{~min}$, permeabilized with $0.2 \%$ Triton $\mathrm{X}-100$ at room temperature for $15 \mathrm{~min}$ and then stained with $0.1 \%$ crystal violet for $5 \mathrm{~min}$. The number of cells on the lower surface, which had invaded through the membrane, was counted under a light microscope in five random fields at a magnification of x100. Data were obtained from three independent experiments. The procedure for the Transwell migration assay was the same as for the Transwell invasion assay, except that the filter of the top chamber was not coated with Matrigel.

Co-cultured cells were also analyzed in 36-mm transwells with $3-\mu \mathrm{m}$ pore polycarbonate membrane inserts (Corning, NY) according to the manufacturer's instructions. The upper and lower cultures were separated by a $3-\mu \mathrm{m}$ pore size polyvinylpyrolidone-free polycarbonate filter. Briefly, the lower chambers were filled with $3 \mathrm{ml}$ of DMEM containing $1 \% \mathrm{FBS}$ and $5 \times 10^{6}$ cells $/ \mathrm{ml}$ in $200 \mu \mathrm{l}$ DMEM/1\% FBS were added to the upper or lower compartment. Chambers were incubated in a humidified $37^{\circ} \mathrm{C}$ atmosphere with $5 \% \mathrm{CO}_{2}$ for $48 \mathrm{~h}$. Cells were then lysed and the proteins were extracted. VEGF and CXCR4 protein expression was detected as detailed below. Supernatants were collected for ELISA assay (3). Each experiment was performed in triplicates.

For CXCR4 blocking, the CXCR4 inhibitor AMD3100 (Sigma-Aldrich), was incubated at a concentration of $10 \mu \mathrm{g} / \mathrm{ml}$ $1 \mathrm{~h}$ before and during the migration and co-culture assays. SDF-1 neutralizing antibody (SDF-1 Ab, $0.6 \mu \mathrm{g} / \mathrm{ml}$ ) was used to specify neutralize SDF-1 (R\&D Systems).

MTT assay. For assessment of cell proliferation, the 3-(4,5-dimethylthiazol-2-yl)-2,5-diphenyltetrazolium bromide (MTT) assay was performed according to the manufacturer's instructions (Sigma-Aldrich). The experiments were repeated three times independently. Anti-human VEGF neutralizing 
A

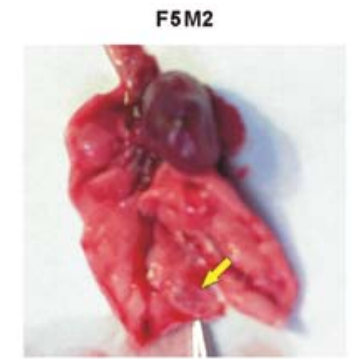

B

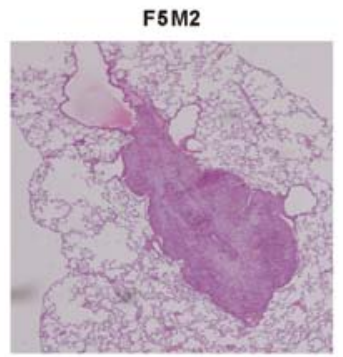

C



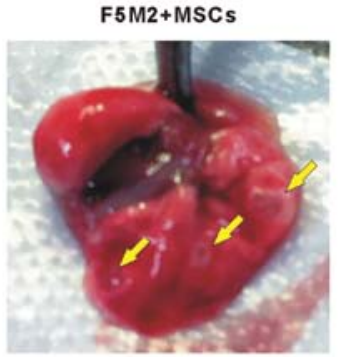

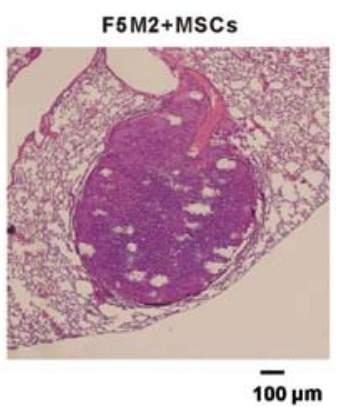

D

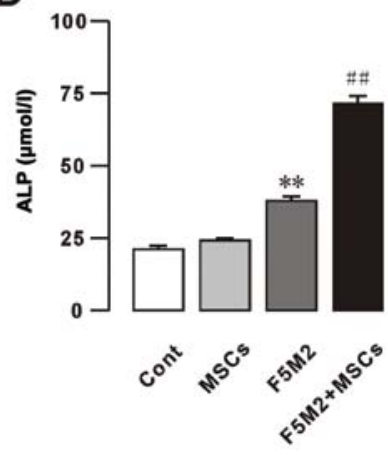

Figure 1. Pulmonary metastasis of F5M2 in vivo in response to co-injection with MSCs. (A) General observation of F5M2 pulmonary metastasis. (B) Pathological analysis (H\&E staining). (C) Nodules per lung. (D) ALP levels in the blood. ${ }^{* *} \mathrm{P}<0.01$, compared to the control group. ${ }^{\text {\# }} \mathrm{P}<0.05$, ${ }^{\# \#} \mathrm{P}<0.01$, compared to the F5M2 group. Black arrows, sites of metastasis nodules. Results are expressed as means \pm SD $(n=6)$.

antibody (VEGF Ab, $0.1 \mu \mathrm{g} / \mathrm{ml}$ ) was used to specify neutralized VEGF (Abcam, USA).

Immunohistochemistry. Cell slices were treated with $3 \%$ hydrogen peroxide in methanol for $10 \mathrm{~min}$, in order to inactivate endogenous peroxidases, and were then incubated with primary antibodies against VEGF or CXCR4 (Abcam) overnight at $4^{\circ} \mathrm{C}$. After rinsing with PBS, cells on the slices were treated for $20 \mathrm{~min}$ with pre-diluted biotin-conjugated broad-spectrum IgG secondary antibody (Gene Tech, China) and then visualized using streptavidin-conjugated horseradish peroxidase provided with the Real Envision detection kit (Gene Tech), following the manufacturer's instructions. The experiments were repeated three times independently.

ELISA assay. To determine the levels of VEGF secreted in the CM from F5M2 cells or MSCs, cells were plated in medium containing $1 \%$ FBS. After cells reached confluence, supernatants were collected according to the manufacturer's instructions. Media were analyzed by a commercially available sandwich VEGF ELISA kit (Invitrogen), according to the manufacturer's instructions. Assays were performed in quadruplicates and the results were normalized for the number of producing cells and reported as picograms (pg) of VEGF per $1 \times 10^{6}$ cells per $48 \mathrm{~h}$.

Western blotting. VEGF and CXCR4 protein expression was detected by western blotting (13). As mentioned above, cells were lysed and proteins were extracted. Protein samples were subjected to sodium dodecyl sulfate polyacrylamide gel eletrophoresis (SDS-PAGE) and transferred to polyvinylidene difluoride membranes by a semi-dry transfer cell system (BioRad, Hercules, CA, USA). The membrane was probed with mouse monoclonal antibodies against VEGF, CXCR4 and $\beta$-actin (internal control) (Abcam).

Statistical analysis. Data were obtained from at least three independent experiments and presented as means \pm SD. Comparisons between two groups were performed with the Student's t-test, while statistical significance of mean differences among multiple groups were obtained by the analysis of variance (ANOVA) followed by Dunnett's post-hoc test. A $\mathrm{P}$-value $<0.05$ was considered as statistically significant.

\section{Results}

MSCs promote osteosarcoma pulmonary metastasis. To study the osteosarcoma interaction with MSCs on pulmonary metastasis, we monitored the development of osteosarcoma pulmonary metastasis in response to MSCs. Six weeks after injection of F5M2 cells, with or without MSCs, into the caudal vein, mice were sacrificed and the total number of osteosarcoma pulmonary metastatic nodules per lung was calculated. A remarkably higher number of tumor nodules was observed after co-injection of F5M2 cells with MSCs compared to F5M2 alone, as confirmed by histological examination of the tumors (Fig. 1A-C). No metastases were found in the liver, spleen or kidney (data not shown). Levels of alkaline phosphatase (ALP) in blood serum were measured at week 6 to determine the progression of osteosarcoma metastasis (2). A statistically significant increase of ALP levels was observed in response to exogenous MSCs compared to the osteosarcoma group (Fig. 1D). Six weeks after injection of Dil-labeled MSCs, we found a large number of MSCs in the lung, which correlated with an enhanced osteosarcoma pulmonary metastasis (Fig. 2). We postulate that F5M2 cells were induced to undergo pulmonary metastasis by components secreted by MSCs or F5M2 cells, which could chemoattract each other, leading to an intricate interaction between them and ultimately to pulmonary metastasis.

MSCs enhance F5M2 cell migration in a co-culture model system. To test whether MSCs have an impact on osteosarcoma cell migration, we initially performed migration and co-culture assays in which MSCs were plated in the bottom chamber of a Corning chamber system. Our results showed that the effect of MSCs on F5M2 cell migration was significantly higher than that of $1 \% \mathrm{FBS}$. In addition, CXCR4 receptor inhibitor AMD3100 $(10 \mu \mathrm{g} / \mathrm{ml})$ decreased the effect of MSCs on F5M2 cell migration (Fig. 3). SDF1-neutralizing antibody (SDF-1 Ab, $0.6 \mathrm{mg} / \mathrm{ml}$ ) also significantly inhibited the F5M2 cell migration (Fig. 3). 


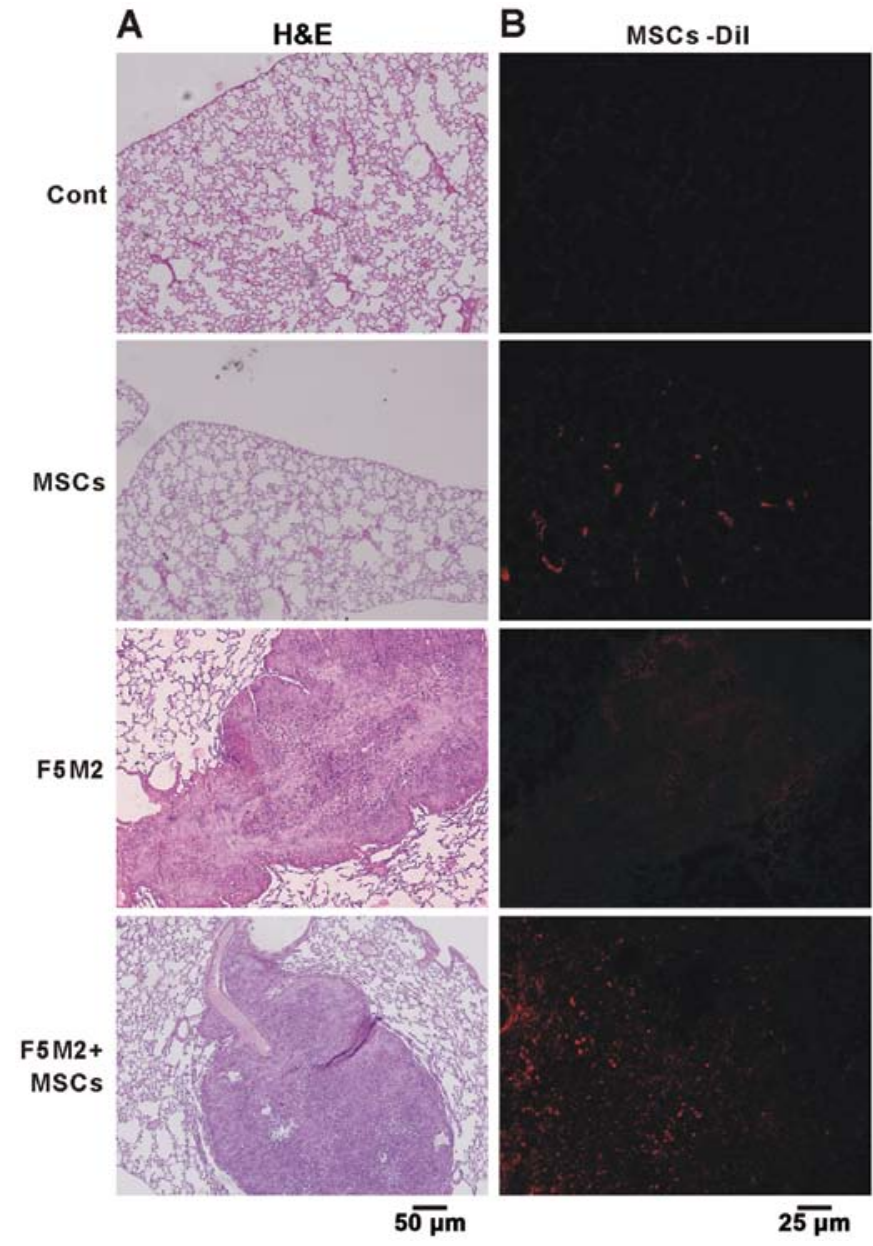

Figure 2. Pulmonary metastasis of F5M2 in response to co-injection with Dilpositive MSCs. (A) Pathological analysis (H\&E staining). (B) Fluorescence microscopy images. Red fluorescence, MSCs labeled with Dil.

F5M2 and MSCs cells were co-cultured as showed in Fig. 4A. In the left and right panel of Fig. 4A, the MSCs or F5M2 cells showed different cell shape and nucleus size stained by hematoxylin. In Fig. 4B, Dil-labeled MSCs were co-cultured with F5M2 cells. The F5M2 single cell or cell clusters migrated from the F5M2 cell cluster to the MSCs (Fig. 4B). Both MSCs and F5M2 cell nucleus stained by Hoechst 33258 showed blue cell nucleus, while the membrane of Dil-labeled MSCs were red. The bright arrow indicates the migrating F5M2 cells from F5M2 cell cluster to MSC cluster (Fig. 4A and B). These results showed that F5M2 cells were chemoattracted by MSCs and migrated towards them.

Enhanced VEGF expression by MSCs in the F5M2-MSCs co-culture system. To study the possible mechanisms underlying the interaction between F5M2 and MSCs, we focused our attention on the CXCR4/VEGF signaling pathway, which is involved in the progression of many cancers (14-17). We analyzed the expression of VEGF and CXCR4 in F5M2 cells and MSCs by immunohistochemistry (Fig. 5A) and western blotting (Fig. 5B and C). To assess the role of VEGF and CXCR4 in the migration of osteosarcoma to MSC sites, we performed Transwell assays using MSCs and CM from F5M2 cells in combination with the CXCR4 receptor inhibitor AMD3100 $(10 \mu \mathrm{g} / \mathrm{ml})$. In the F5M2-MSC co-culture model
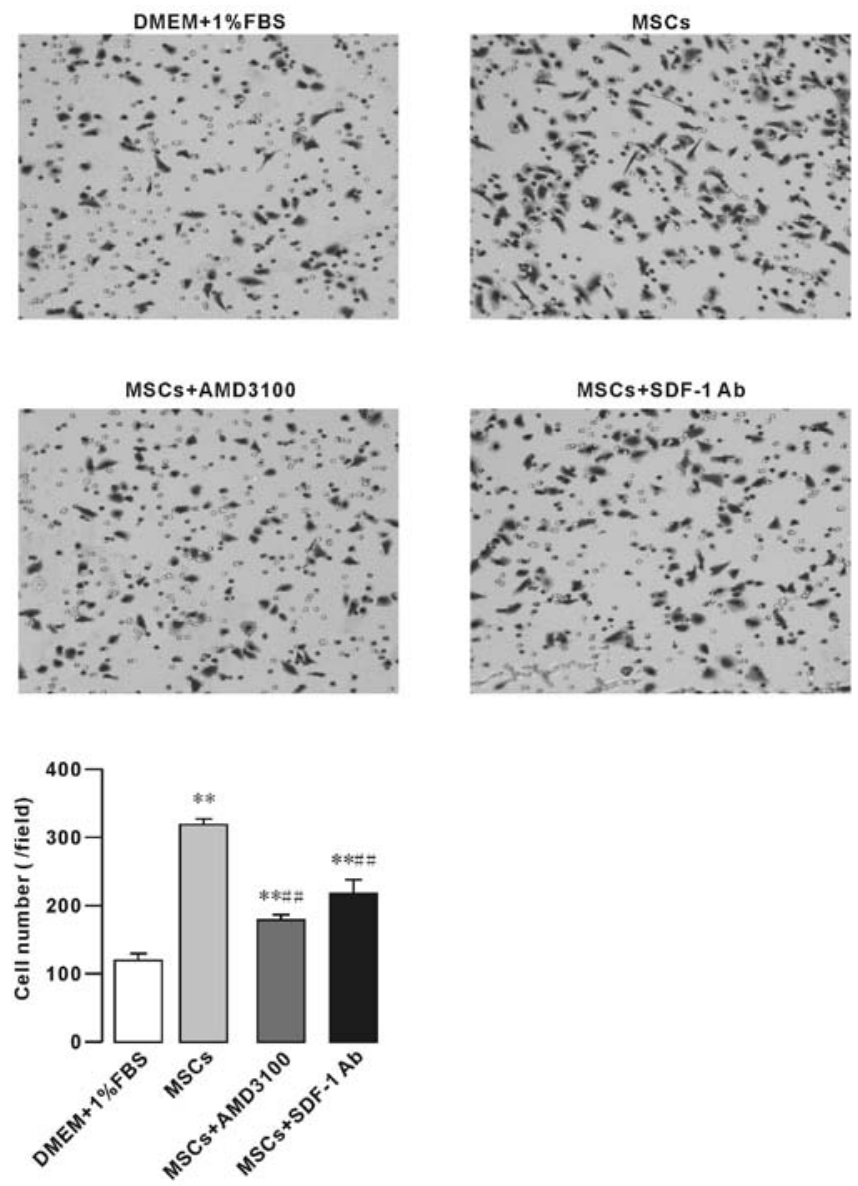

Figure 3. Migration of F5M2 towards MSCs. Transwell assays were performed in triplicates. Cells were counted by light microscopy and the mean cell number of five fields of each well was used for statistics. Results were expressed as means $\pm \mathrm{SD} .{ }^{* *} \mathrm{P}<0.01$, compared to the DMEM $+1 \%$ FBS group. ${ }^{\# \#} \mathrm{P}<0.01$, compared to the MSC group.

system, positive immunohistochemical stainings for VEGF and CXCR4 are showed in Fig. 5A. VEGF protein was detected in the cytoplasm and membrane of MSCs. We observed that the low basal VEGF expression of MSCs increased when MSCs were co-cultured with F5M2 CM, using either Transwell chambers or direct contact with F5M2 cells for $48 \mathrm{~h}$. We also found that the expression of VEGF was alleviated by the use of the CXCR4 receptor inhibitor AMD3100 $(10 \mu \mathrm{g} / \mathrm{ml})$ in the MSCs plus F5M2 group. The VEGF expression of F5M2 was not different in the three groups (F5M2, Transwell and direct co-culture groups, data not shown).

CXCR4 expression was detected in the nucleus as well as in the cytoplasm of F5M2 cells. CXCR4 expression of F5M2 cells was not significantly increased in the F5M2+MSCs group compared to the F5M2 group and in the presence of exogenous VEGF $(100 \mathrm{ng} / \mathrm{ml})$. In addition, the CXCR4 expression of MSCs was not different in the three groups (MSCs, Transwell and direct co-culture groups, data not shown).

VEGF secretion from MSCs is modulated by F5M2. We measured the levels of VEGF secreted by MSCs and F5M2 by ELISA (Fig. 6A and B) in the following groups: F5M2 cells $\left(1.0 \times 10^{6}\right.$ cells) alone, MSCs $\left(1.0 \times 10^{6}\right.$ cells) alone, co-culture 

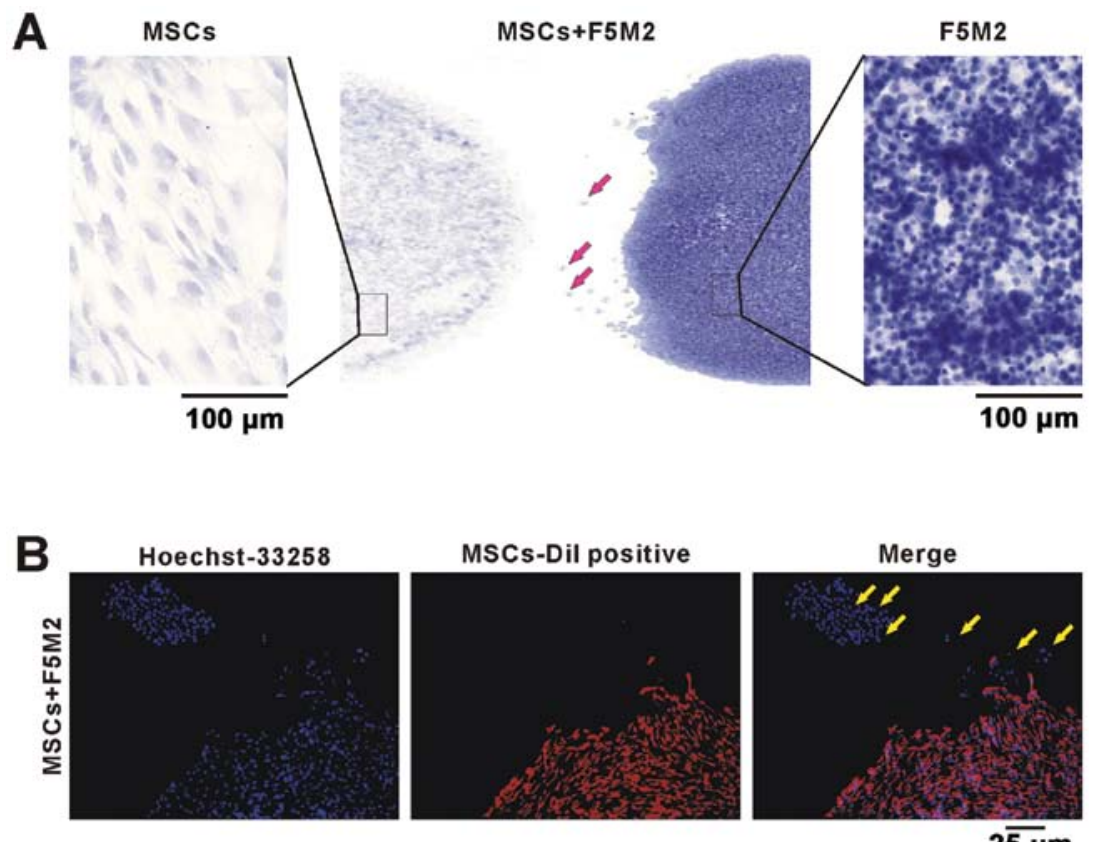

Figure 4. Migration of F5M2 cells towards MSCs in a co-culture system. (A) Representatively histological staining (hematoxylin) of co-cultured F5M2 cells and MSCs, the bright arrow indicate that the F5M2 cell or cell cluster migrate towards MSCs. (B) Dil-stained (red) MSCs and Hoechst-stained F5M2 and MSC cells in co-culture system.

of F5M2 cells and MSCs $\left(0.5 \times 10^{6}\right.$ cells each $)$ and F5M2 cells treated with the CXCR4 receptor inhibitor AMD3100. MSCs secreted very low levels of VEGF (Fig. 6A). We subtracted from the amount of VEGF, in the final supernatant of MSCs cultured in the conditioned medium. However, MSCs co-cultured with F5M2 secreted higher levels of VEGF than the F5M2 or MSC groups (Fig. 6A). On the other hand, the F5M2 plus MSC Transwell, or mixed group, secreted higher VEGF levels, which were dramatically decreased upon treatment with AMD3100 (10 $\mu \mathrm{g} / \mathrm{ml})$ (Fig. 6B).

We further studied the VEGF expression on the metastatic tumor in vivo, positive immunohistochemical stainings for VEGF was found in the plumonary metastasis sites of the nude mice. We found VEGF higher expressed in the tumor site as showed in Fig. 6C, and the VEGF-positive staining was not statistically different between the F5M2 and F5M2+MSCs groups. We also checked the serum VEGF concentration in the control, MSCs, F5M2 and F5M2+MSCs groups, and found the VEGF concentration was higher in the F5M2 and F5M2+MSCs groups compared with the control group, especially in the F5M2+MSCs group the VEGF concentration was significantly higher than the F5M2 group as showed in Fig. 6D.

VEGF promotes proliferation of F5M2 cells and MSCs. To assess the possible role of VEGF on F5M2 and MSC growth, we performed MTT assays on cells stimulated with exogenous VEGF (100 ng/ml). The results showed that VEGF significantly increased the proliferation of F5M2 cells and MSCs (Fig. 7A and B). After 3 days, the number of MSCs in the presence of VEGF was $\sim 20 \%$ higher than that in its absence. Similarly, after 2 days, the number of F5M2 cells in the presence of VEGF was $\sim 10 \%$ higher than that in its absence. These data show that
VEGF modulates the proliferation of both F5M2 and MSCs. Furthermore, we found the proliferation of F5M2 cells were actually promoted in the co-culture condition, moreover we also used the VEGF neutralizing antibody (VEGF Ab, $0.1 \mu \mathrm{g}$ / $\mathrm{ml})$ to verify the VEGF effect on the OS cell proliferation ability, and found the VEGF Ab could significantly inhibit the F5M2 cell proliferation by $\sim 30 \%$ as showed in Fig. 7C.

\section{Discussion}

Osteosarcoma is the most frequent primary bone tumor in young adults and adolescents. Pulmonary metastasis occurs in $\sim 40-50 \%$ of the osteosarcoma patients (18), who present an overall 5-year survival rate of only $28 \%$ (14). CXCR4 and its ligand, stromal cell-derived factor 1 (SDF-1), has been found highly expressed in various cancer and play important roles in tumorigenicity, proliferation, metastasis and angiogenesis (19-24). It has been shown that CXCR4 also plays an important role in the migration of hematopoietic stem cells and downstream progenitors from, and their retention within, the bone marrow. On the other hand, CXCR4 inhibitors may be used to inhibit tumor growth and metastasis and also to mobilize hematopoietic stem cells from the bone marrow into circulation (7).

However, the underlying mechanisms under which MSCs target osteosarcoma sites and promote osteosarcoma cell growth and pulmonary metastasis are still unclear. It has been demonstrated that MSCs within tumor stroma promote metastasis (8). In our study, we found that MSCs were involved in the emergence and growth of osteosarcoma pulmonary metastasis (Fig. 2). An enhanced tumor cell number was observed in response to the injection of MSCs and F5M2 compared to F5M2 alone. In our experiments, F5M2 single 

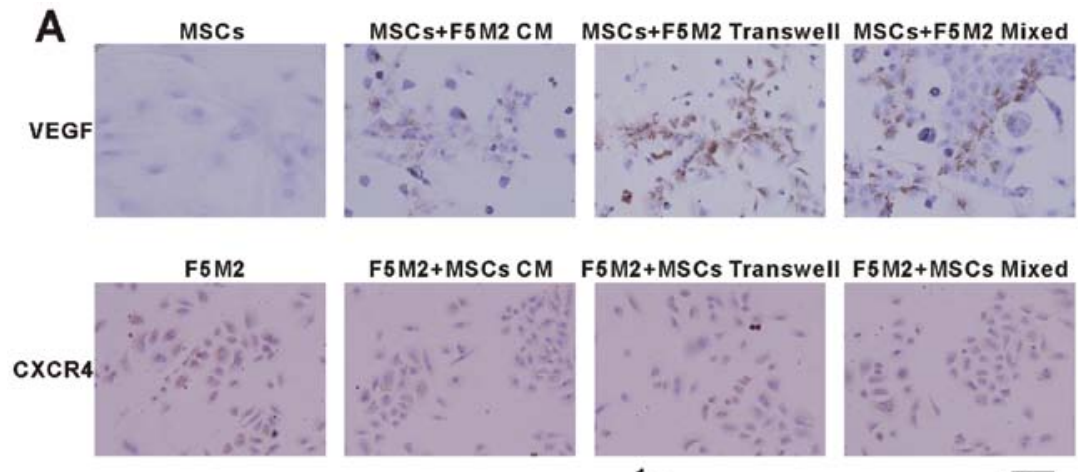

F5 M2+MSCs CM F5M2+MSCs Transwell F5M2+MSCs Mixed
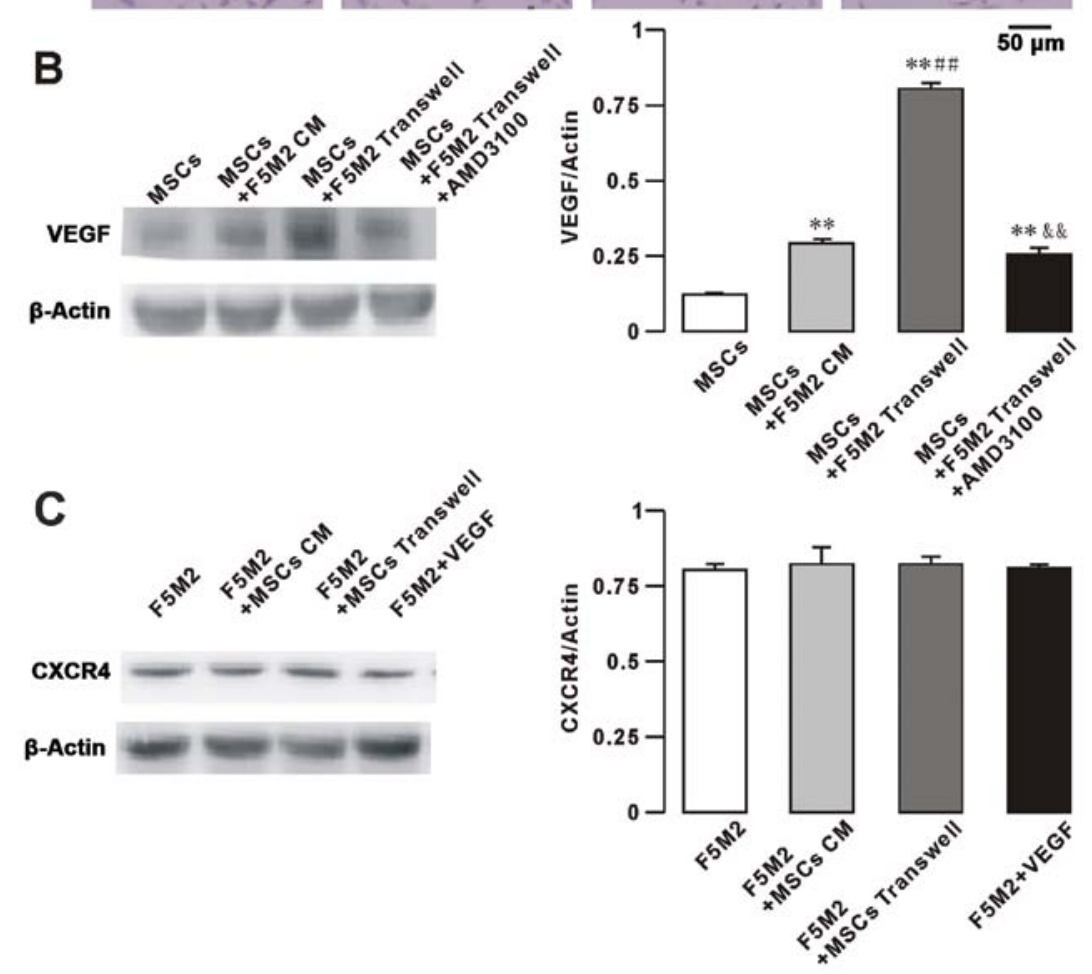

Figure 5. VEGF and CXCR4 protein expression in MSCs and F5M2 in a co-culture model system. (A) Immunohistochemistry for VEGF and CXCR4. (B) Western blotting for VEGF expression of MSCs. Results are expressed as means \pm SD. ${ }^{* *} \mathrm{P}<0.01$, compared to the MSCs group. ${ }^{\# \#} \mathrm{P}<0.01$, compared to the MSCs+F5M2 CM group. \&\& $\mathrm{P}<0.01$, compared to the MSCs+F5M2 Transwell group ( $\mathrm{n}=3$ ). (C) Western blotting for CXCR4 expression of F5M2. Results are expressed as means $\pm \mathrm{SD},(\mathrm{n}=3)$. There are no significant differences between the F5M2, F5M2+MSCs, MSCs+F5M2 Transwell and F5M2+VEGF groups.

cell or F5M2 cell clusters migrated from the F5M2 cell cluster to the MSCs cluster (Fig. 4A and B). Tumor-targeting properties of stem cells have been previously reported (25) and also in studies of tumor angiogenesis with hematopoietic stem cells (26). Xu et al showed the human mesenchymal stem cells (hMSCs) target osteosarcoma and promote its growth and pulmonary metastasis (27). The study of Xu et al (27) indicated SDF-1 and CCL5 played essential roles in the interaction between hMSCs and OS. Our data showed that MSCs promote osteosarcoma pulmonary metastasis. The mechanisms involved in VEGF from MSCs could promote the OS growth. In addition, CXCR4 plays an important role in regulating the VEGF of MSCs. A remarkable accumulation of MSCs in pulmonary metastatic sites was observed after injection with F5M2 cells through the caudal vein of mice. Furthermore, we also provide evidence that the migration of F5M2 was promoted in response to MSCs in the co-culture model system, which was decreased upon treatment with AMD3100 (Fig. 3).
The expression of CXCR4 has been shown to be regulated by VEGF (28). VEGF is also a known inducer of osteosarcoma angiogenesis which could determine the vascular endothelial cell activation, proliferation and migration (29). VEGF induced the expression of CXCR4 via an autocrine pathway (13). The study also showed that CXCR4 inhibition could reduce angiogenesis by inhibiting osteosarcoma neovessel formation as well as cell migration and invasion by decreasing the expression of conventional proangiogenic VEGFA165 $(30,31)$. Patients whose tumors exhibited a positive VEGF165 expression presented poor prognosis (11). The study also found a significant correlation between the expression of CXCR4 and VEGF in osteosarcoma samples (32), which suggested that the CXCR4/VEGF could be a predictor of potential metastatic development in osteosarcoma (10). Our data revealed that MSCs secrete very low levels of VEGF, but with F5M2 conditioned medium the MSCs secreted higher levels of VEGF (Fig. 6A). On the other hand, F5M2 plus MSCs Transwell or mixed group secreted higher VEGF 
A



C

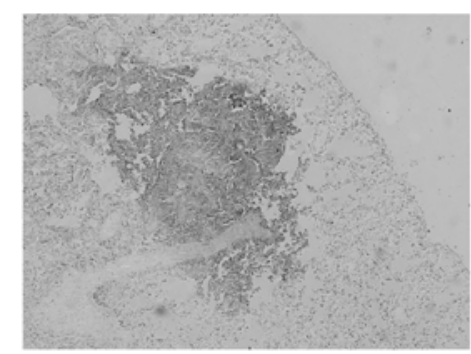

B

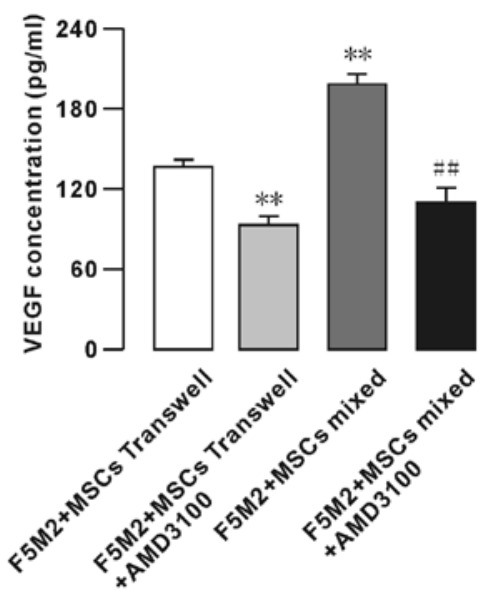

D

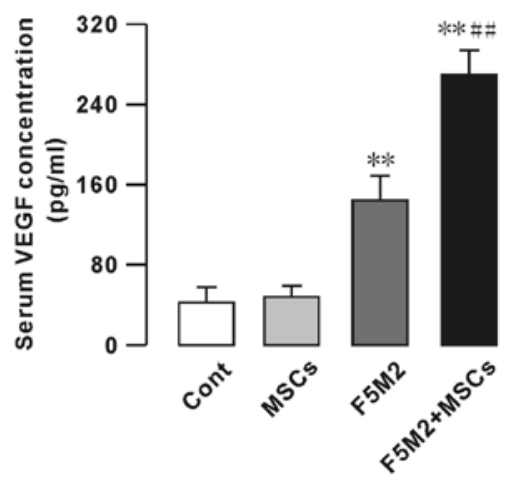

Figure 6. VEGF secretion in MSCs and F5M2 cells co-culture system and serum VEGF concentration of xenografts of nude mice. Supernatants were assayed for VEGF content by ELISA, AMD3100 $(10 \mu \mathrm{g} / \mathrm{ml})$ was added $1 \mathrm{~h}$ before and during the co-culture assay. Results were normalized for the number of producing cells and reported as picograms (pg) of VEGF per $1 \times 10^{6}$ cells after $48 \mathrm{~h}$ and expressed as means $\pm \mathrm{SD},(\mathrm{n}=3)$. (A) VEGF secretion in MSCs and F5M2. ${ }^{* *} \mathrm{P}<0.01$, compared to the MSCs group. (B) VEGF secretion in MSCs+F5M2 cells. ${ }^{* *} \mathrm{P}<0.01$, compared to the F5M2+MSCs group. ${ }^{\# \#} \mathrm{P}<0.01$, compared to the F5M2+MSCs mixed group. (C) Representative positive VEGF immunohistochemistry staining of pulmonary metastatic tumor. (D) Serum VEGF concentration of mouse xenografts. ${ }^{* *} \mathrm{P}<0.01$, compared to the control group. ${ }^{\# \#} \mathrm{P}<0.01$, compared to the F5M2 group. Results are expressed as means $\pm \mathrm{SD}$, (n=3).

A

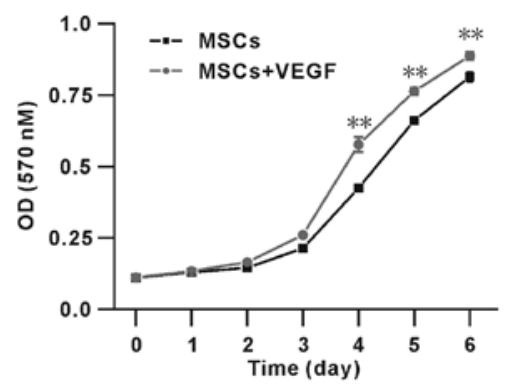

C



B



Figure 7. Effects of VEGF on MSCs and F5M2 cell proliferation. (A) Effects of VEGF (100 ng/ml) on MSC growth. Cell proliferation was measured at the indicated time points using the MTT assay as described in Materials and methods ( $\mathrm{n}=3$; $\left.{ }^{* *} \mathrm{P}<0.01\right)$. (B) Effects of VEGF $(100 \mathrm{ng} / \mathrm{ml})$ on F5M2 cell growth. $(\mathrm{n}=3$; $\left.{ }^{* *} \mathrm{P}<0.01\right)$. (C) Cell proliferation of F5M2 and the effects of VEGF Ab $(0.1 \mu \mathrm{g} / \mathrm{ml})$ on F5M2 cell growth in mixed culture CM. $\left(\mathrm{n}=3 ;{ }^{* * *} \mathrm{P}<0.01\right)$. 
levels than the individual cell groups, which were dramatically decreased upon treatment with the CXCR4 inhibitor AMD3100 $(10 \mu \mathrm{g} / \mathrm{ml})$ (Fig. 6B). These results showed that CXCR4 modulates the production of VEGF in MSCs. Co-cultured with F5M2 or treated with F5M2-conditioned medium, MSCs expressed and secreted higher VEGF levels. CXCR4 expression on F5M2 cells was, however, not significantly increased in the co-culture model (Fig. 5B).

CXCR plays a central role in determining the target organ where tumor cells metastasize $(1,33)$, as well as the blood supply via angiogenesis $(30,34)$. CXCLs released from the endothelium of target tissues (i.e., lung and bone) bind to their receptors, CXCRs on tumor cells, which enables the metastasis to the specific target tissues. Studies have indicated that CXCR4 plays a role in regulating metastasis of many solid tumors and their neovasculature (10,35-38). Moreover, there is a correlation between the expression of CXCR4/VEGF and blood-borne metastasis (35). Our data show that MSCs secreted higher VEGF levels when co-cultured with F5M2 cells or transplanted with F5M2 cells in vivo (Fig. 6). In vitro study we also found VEGF could increase the growth of both F5M2 cells and MSCs.

Taken together, our data showed that MSCs interacted with osteosarcoma cells and promoted osteosarcoma pulmonary metastasis, in which the expression of CXCR4 by osteosarcoma cells modulated the expression and secretion of VEGF by MSCs. It suggested that CXCR4 mediated osteosarcoma growth and pulmonary metastasis were promoted by MSCs through VEGF.

\section{Acknowledgements}

This study was supported by grants from the National Nature Science Foundation of China (no. 30800432 to L.D. and no. 81072194 to B.-A.M.). We would like to thank Dong Shuhua, the English editor, from Guangdong University of Foreign Studies.

\section{References}

1. Murphey MD, Robbin MR, McRae GA, Flemming DJ, Temple HT and Kransdorf MJ: The many faces of osteosarcoma. Radiographics 17: 1205-1231, 1997.

2. Link MP, Goorin AM, Miser AW, et al: The effect of adjuvant chemotherapy on relapse-free survival in patients with osteosarcoma of the extremity. N Engl J Med 314: 1600-1606, 1986.

3. Chen X, Yang TT, Wang W, et al: Establishment and characterization of human osteosarcoma cell lines with different pulmonary metastatic potentials. Cytotechnology 61: 37-44, 2009.

4. Friedenstein AJ, Chailakhyan RK, Latsinik NV, Panasyuk AF and Keiliss-Borok IV: Stromal cells responsible for transferring the microenvironment of the hemopoietic tissues. Cloning in vitro and retransplantation in vivo. Transplantation 17 : 331-340, 1974

5. Austin TW, Solar GP, Ziegler FC, Liem L and Matthews W: A role for the Wnt gene family in hematopoiesis: expansion of multilineage progenitor cells. Blood 89: 3624-3635, 1997.

6. Dexter TM, Spooncer E, Schofield R, Lord BI and Simmons P: Haemopoietic stem cells and the problem of self-renewal. Blood Cells 10: 315-339, 1984.

7. Reya T, Duncan AW, Ailles L, et al: A role for Wnt signalling in self-renewal of haematopoietic stem cells. Nature 423: 409-414, 2003 .

8. Karnoub AE, Dash AB, Vo AP, et al: Mesenchymal stem cells within tumour stroma promote breast cancer metastasis. Nature 449: 557-563, 2007.
9. Coussens LM and Werb Z: Inflammation and cancer. Nature 420: 860-867, 2002.

10. Oda Y, Yamamoto H, Tamiya S, et al: CXCR4 and VEGF expression in the primary site and the metastatic site of human osteosarcoma: analysis within a group of patients, all of whom developed lung metastasis. Mod Pathol 19: 738-745, 2006.

11. Li D, Dai K and Tang T: Effects of dextran on proliferation and osteogenic differentiation of human bone marrow-derived mesenchymal stromal cells. Cytotherapy 10: 587-596, 2008.

12. Pittenger MF, Mackay AM, Beck SC, et al: Multilineage potential of adult human mesenchymal stem cells. Science 284: 143-147, 1999.

13. Liang $\mathrm{Z}, \mathrm{Wu} \mathrm{T}$, Lou $\mathrm{H}$, et al: Inhibition of breast cancer metastasis by selective synthetic polypeptide against CXCR4. Cancer Res 64: 4302-4308, 2004.

14. Kempf-Bielack B, Bielack SS, Jurgens H, et al: Osteosarcoma relapse after combined modality therapy: an analysis of unselected patients in the Cooperative Osteosarcoma Study Group (COSS). J Clin Oncol 23: 559-568, 2005.

15. Kim J, Takeuchi H, Lam ST, et al: Chemokine receptor CXCR4 expression in colorectal cancer patients increases the risk for recurrence and for poor survival. J Clin Oncol 23: 2744-2753, 2005.

16. Retz MM, Sidhu SS, Blaveri E, et al: CXCR4 expression reflects tumor progression and regulates motility of bladder cancer cells. Int J Cancer 114: 182-189, 2005.

17. Russell HV, Hicks J, Okcu MF and Nuchtern JG: CXCR4 expression in neuroblastoma primary tumors is associated with clinical presentation of bone and bone marrow metastases. J Pediatr Surg 39: 1506-1511, 2004.

18. Wada T, Isu K, Takeda N, Usui M, Ishii S and Yamawaki S: A preliminary report of neoadjuvant chemotherapy NSH-7 study in osteosarcoma: preoperative salvage chemotherapy based on clinical tumor response and the use of granulocyte colonystimulating factor. Oncology 53: 221-227, 1996.

19. Beckermann BM, Kallifatidis G, Groth A, et al: VEGF expression by mesenchymal stem cells contributes to angiogenesis in pancreatic carcinoma. Br J Cancer 99: 622-631, 2008.

20. Djouad F, Plence P, Bony C, et al: Immunosuppressive effect of mesenchymal stem cells favors tumor growth in allogeneic animals. Blood 102: 3837-3844, 2003.

21. Gunn WG, Conley A, Deininger L, Olson SD, Prockop DJ and Gregory CA: A crosstalk between myeloma cells and marrow stromal cells stimulates production of DKK1 and interleukin-6: a potential role in the development of lytic bone disease and tumor progression in multiple myeloma. Stem Cells 24: 986-991, 2006.

22. Otsu K, Das S, Houser SD, Quadri SK, Bhattacharya S and Bhattacharya J: Concentration-dependent inhibition of angiogenesis by mesenchymal stem cells. Blood 113: 4197-4205, 2009.

23. Potier E, Ferreira E, Andriamanalijaona R, et al: Hypoxia affects mesenchymal stromal cell osteogenic differentiation and angiogenic factor expression. Bone 40: 1078-1087, 2007.

24. Romieu-Mourez R, Francois M, Boivin MN, Bouchentouf M, Spaner DE and Galipeau J: Cytokine modulation of TLR expression and activation in mesenchymal stromal cells leads to a proinflammatory phenotype. J Immunol 182: 7963-7973, 2009.

25. Aboody KS, Brown A, Rainov NG, et al: Neural stem cells display extensive tropism for pathology in adult brain: evidence from intracranial gliomas. Proc Natl Acad Sci USA 97: 12846-12851, 2000.

26. De Palma M, Venneri MA, Roca $\mathrm{C}$ and Naldini L: Targeting exogenous genes to tumor angiogenesis by transplantation of genetically modified hematopoietic stem cells. Nat Med 9: 789-795, 2003.

27. Xu WT, Bian ZY, Fan QM, Li G and Tang TT: Human mesenchymal stem cells (hMSCs) target osteosarcoma and promote its growth and pulmonary metastasis. Cancer Lett 281: 32-41, 2009.

28. Bachelder RE, Wendt MA and Mercurio AM: Vascular endothelial growth factor promotes breast carcinoma invasion in an autocrine manner by regulating the chemokine receptor CXCR4. Cancer Res 62: 7203-7206, 2002.

29. Plate K: From angiogenesis to lymphangiogenesis. Nat Med 7: 151-152, 2001.

30. de Nigris F, Balestrieri ML, Williams-Ignarro S, et al: Therapeutic effects of autologous bone marrow cells and metabolic intervention in the ischemic hindlimb of spontaneously hypertensive rats involve reduced cell senescence and CXCR4/Akt/eNOS pathways. J Cardiovasc Pharmacol 50: 424-433, 2007. 
31. de Nigris F, Crudele V, Giovane A, et al: CXCR4/YY1 inhibition impairs VEGF network and angiogenesis during malignancy. Proc Natl Acad Sci USA 107: 14484-14489, 2010.

32. Lin F, Zheng SE, Shen Z, et al: Relationships between levels of CXCR4 and VEGF and blood-borne metastasis and survival in patients with osteosarcoma. Med Oncol 28: 649-653, 2011.

33. Balkwill F: The significance of cancer cell expression of the chemokine receptor CXCR4. Semin Cancer Biol 14: 171-179, 2004.

34. Busillo JM and Benovic JL: Regulation of CXCR4 signaling. Biochim Biophys Acta 1768: 952-963, 2007.

35. Laverdiere C, Hoang BH, Yang R, et al: Messenger RNA expression levels of CXCR4 correlate with metastatic behavior and outcome in patients with osteosarcoma. Clin Cancer Res 11: 2561-2567, 2005.
36. Lee YH, Tokunaga T, Oshika Y, et al: Cell-retained isoforms of vascular endothelial growth factor (VEGF) are correlated with poor prognosis in osteosarcoma. Eur J Cancer 35: 1089-1093, 1999.

37. Murakami T, Maki W, Cardones AR, et al: Expression of CXC chemokine receptor- 4 enhances the pulmonary metastatic potential of murine B16 melanoma cells. Cancer Res 62: 7328-7334, 2002

38. Perissinotto E, Cavalloni G, Leone F, et al: Involvement of chemokine receptor 4/stromal cell-derived factor 1 system during osteosarcoma tumor progression. Clin Cancer Res 11: 490-497, 2005. 